\title{
Degenerative Joint Abnormalities in Patients with Paraplegia of Duration Greater than 20 Years
}

\author{
E. J. Wylie, MD, MB BS, DRACR, T. M. H. Chakera, MD, FRCP, FRCR, \\ FRACR \\ Department of Diagnostic Radiology, Royal Perth (Rehabilitation) Hospital, Selby \\ Street, Shenton Park, Western Australia, 6009.
}

\section{Summary}

Radiographs and medical and surgical records of 51 patients following spinal injury have been reviewed. All the patients had been paraplegic for 20 years or more at the time of the survey. The reason for the survey was to assess the incidence of degenerative change of the hips, sacroiliac and shoulder joints in patients following spinal injury. Our review indicates that the number of paraplegic patients who develop degeneration of hip and sacroiliac joint changes is less significant than has been previously reported. Analysis of our figures has revealed certain important associations which predispose these patients to joint degeneration. The associations (anatomical level of injury, urinary tract infections, and the level of the patients' activity) with joint degeneration are described.

Key words: Paraplegia; Foint degeneration; Urinary tract infections.

An association has been suggested between the anatomical level of the patient's paraplegia with the frequency and severity of sacroiliac joint changes (Khan et al., 1979). Similarly, urinary infection in paraplegic patients has been reported to affect the frequency and severity of sacro-iliac joint changes. (Khan et al., 1979). Reduced patient mobility has been described in association with hip joint narrowing (Pool, 1974) and sacro-iliac joint changes (Khan et al., 1979). In view of the published findings we have chosen to report on the correlation between degenerative joint changes and the association between:-

1. Anatomical level of the injury

2. Urinary tract infection

3. Level of patient's activity

\section{Patients and methods}

We studied 51 patients, 44 males and 7 females, all patients except one having 
sustained a traumatic paraplegia; the exception was a patient who developed post-operative paraplegia following surgery for a thoracic spinal arterio-venous malformation. All patients are attending the Royal Perth (Rehabilitation) Hospital as outpatients. The major factors thought to play a part in the development of joint abnormalities were assessed as follows:-

1. Anatomical level of the injury.

2. The patient's history of urinary infection was obtained from the urological review notes, where regular urine analysis and culture was performed. The patient's findings were then divided into three catagories:-

a. Rare or occasional urinary tract infections

b. Infrequent-included patients with occasional severe infections over 20 year span, requiring antibiotic therapy.

c. Frequent/severe-patients who have recurrent persistent infection often requiring treatment, frequently with hospital admission for septicaemia. Those patients with permanent in-dwelling catheters were included in the third group.

3. The level of patient activity was determined by the physiotherapists at the Rehabilitation Hospital. Activity levels were graded as follows:-

a. Active (including patients involved in regular sporting activity and those walking on crutches and calipers.

b. Moderately active (included those in fulltime employment and those with manual wheelchairs.

c. Inactive category included those sedentary people and those with electric wheelchairs

The radiographic assessment of the joints was predominantly from the routine follow-up intravenous pyelogram films (IVPs are performed regularly at 2-3 yearly intervals in these patients). The hip joint measurements were taken from the femoral head to the acetabular roof and the range of normality of 3-5 $\mathrm{mm}$ as described by Fredensberg (1978) was adopted. Sacro-iliac joints were assessed from these same radiographs. The shoulder joints were assessed most frequently from the patients' chest radiographs taken as part of the investigation for intermittent chest infections and other medical conditions. The radiographs were graded as follows:

a. Normal-no pathological change detected.

b. Early-Joint space narrowing (less than $3 \mathrm{~mm}$ ).

c. Moderate-Joint space narrowing, osteophytes and subchondral sclerosis.

d. Severe-Above changes plus subarticular cysts and loose bodies.

\section{Results}

51 patients who had been paraplegic for more than 20 years at the time of examination in 1985 were reviewed. Seventeen patients had cervical spinal lesions while 26 patients had thoracic and 8 patients had lumbar spinal lesions.

\section{Hip joints}

Of the 51 patients examined, 27 patients had at least 1 abnormal hip joint. 
Table I Anatomical level of spinal injury versus degenerative hip change

\begin{tabular}{lccccc}
\hline & Normal & Early & Moderate & Severe & Total \\
\hline Cervical & 5 & 2 & 7 & 3 & 17 \\
Thoracic & 14 & 11 & 1 & - & 26 \\
Lumbar & 5 & 2 & 1 & - & 8 \\
Total & 24 & 15 & 9 & 3 & 51 \\
\hline
\end{tabular}

Eleven out of $17(70 \%)$ of the patients with cervical injuries developed hip changes while only 3 out of $8(37 \%)$ of lumbar spinal injuries developed degenerative hip changes. Ten out of $12(83 \%)$ of the moderate and severe changes were found in patients with cervical injuries even though cervical injuries only accounted for $33^{\circ}$ o of all injuries.

The above figures indicate that anatomical level of injury is a most important determining factor as regards development of hip joint degeneration. Patients with cervical injury regardless of their muscle tone are more likely to develop hip joint degeneration than those with thoracic or lumbar injury. Inactive muscle bulk is greater in patients with cervical injuries.

Table II Activity levels versus degenerative hip changes

\begin{tabular}{lccccc}
\hline & Normal & Early & Moderate & Severe & Total \\
\hline Active & 13 & 4 & 4 & - & 21 \\
Moderate & 5 & 5 & 3 & 1 & 14 \\
Inactive & 6 & 6 & 2 & 2 & 16 \\
Total & 24 & 15 & 9 & 3 & 51 \\
\hline
\end{tabular}

These figures indicate that the patients who are inactive or have reduced level of activity tend to develop degenerative changes in the hip joints more commonly than do more active patients. Eight out of $21\left(38^{\circ}\right)$ of active patients developed hip changes while 10 out of $16(65 \%)$ of inactive patients developed such changes. No severe degenerative changes were seen in active patients.

We found no signficiant correlation between incidence of urinary tract infections and degenerative changes in the hip joints.

Table III Average age of patients in years with degenerative hip joint changes

\begin{tabular}{cccc}
\hline Normal & Early & Moderate & Severe \\
\hline 45 & 47 & 44 & 39 \\
\hline
\end{tabular}

There was no significant difference in the average age of patients in each category of degenerative changes. Average age of patients in each group is similar. None of the above group is biased to developed degenerative changes due to preponderance of elderly patients in that subgroup of patients.

\section{Sacro-iliac joints}

Only 5 out of the $51(13 \%)$ of patients were shown to have abnormal sacro-iliac joints.

Table IV Anatomical level and activity level versus degenerative SI joint changes

The numbers with degenerative changes are so small that no logical conclusion could be drawn from comparison of the level of spinal injury or the activity level with the severity of degenerative changes. 
Table V Urinary versus degenerative sacro-iliac joint change

\begin{tabular}{lccccc}
\hline & Normal & Early & Moderate & Severe & Total \\
\hline Frequent (severe) & 21 & 1 & - & 1 & 23 \\
Infrequent (moderate) & 14 & 1 & - & 1 & 16 \\
Rare (negligible) & 10 & 2 & - & - & 12 \\
Total & 45 & 4 & - & 2 & 51 \\
\hline
\end{tabular}

These figures indicate no definite relationship between the severity of the infection and the occurrence of sacro-iliac joint abnormalities.

\section{Shoulder joint}

Shoulder radiographs for review were only available for 38 of the 51 patients studied.

Table VI Urinary infections versus degenerative shoulder changes

No correlation was found between shoulder joint degeneration and urinary infection.

Table VII Activity level versus degenerative shoulder changes

\begin{tabular}{lccccc}
\hline & Normal & Early & Moderate & Severe & Total \\
\hline Active & 13 & 2 & 1 & - & 16 \\
Moderate & 7 & 3 & 1 & - & 11 \\
Inactive & 6 & 2 & 1 & 2 & 11 \\
Total & 26 & 7 & 3 & 2 & 38 \\
\hline
\end{tabular}

These figures indicate that shoulder joint degeneration is more likely to occur in the less active patients.

Only 3 out of $13\left(18^{\circ}\right.$ ) of active patients developed degenerative changes while 5 out of $11(45 \%)$ of inactive patients developed shoulder changes

\section{Discussion}

\section{Hip joints}

Poole (1974) previously described the frequent occurrence of hip joint space narrowing in flaccid paresis. He surmised that patients with flaccid paresis develop joint space narrowing due to poor cartilage metabolism. The outer twothirds of the cartilage of the hip joint receives its nutrition from the synovial fluid which is circulated by the pumping action of the surrounding muscles. Joint immobilisation also causes a sharp curtailment in production of synovial fluid further compromising cartilage nutrition.

Our results indicate that cervical and thoracic injury is more frequently associated with hip joint space degeneration. Twelve out of 17 (or $70^{\circ}{ }_{0}$ ) of patients with cervical spinal injury while only 1 out of 8 patients with a lumbar spinal injury developed moderate joint degeneration. No severe degenerative hip joint changes were seen in any patients with thoracic or lumbar spinal injuries. These findings presumably indicate the extent of denervation of muscle mass and therefore the loss of effective muscle pumping contributing to normal synovial fluid circulation and production is an important factor.

Of the two factors (muscle tone and level of injury) contributing to hip joint 
degeneration, we feel that the level of injury is the more important factor; the higher the level the more likely the patient is to develop hip joint degeneration.

Patient activity levels may well reflect similar findings in that only 8 out of 21 $(38 \%)$ of patients judged to be active developed degenerative hip changes as compared to 10 out of $16(62 \%)$ of those considered to be inactive. Presumably these active patients have more passive muscle movement contributing to joint movement and synovial fluid circulation.

\section{Sacro-iliac joints}

Sacro-iliac joint changes have been quoted in 30-61\% of paraplegics (Wright et al., 1965) often described as being similar to those changes found in ankylosing spondylitis (Khan et al., 1979). Only 6 out of 51 (13\%) of our series developed sacro-iliac changes. Speculation about the aetiology of these changes include the association with chronic urinary tract infection because of the high incidence of such infection in paraplegic patients, and the high incidence of ureteritis in Rieter's disease, and ankylosing spondylitis (Anderson, 1981).

Urinary infections were believed to spread to affect the SI joints via the prostatic venous plexuses. Two of our patients were found to have complete ankylosis, while four others developed osteophytes and subchondral sclerosis. No patient developed peri-articular erosions suggestive of ankylosing spondylitis (Khan et al., 1979). The nature of the sacro-iliac changes themselves closely reflect the changes described by Khan (1979). Like Wright (1965) we did not demonstrate changes of sacro-ilitis in any of our patients and we demonstrated no higher incidence of sacro-iliac changes with increased frequency of urinary infection. SI joint abnormalities have been reported to be more frequent and more severe in patients with high levels of spinal injury (Khan et al., 1979). Unfortunately the number of patients who developed sacro-iliac changes in our study was not large enough to draw a logical conclusion about its relation to the level of spinal injury.

The incidence of sacro-iliac joint abnormalities amongst our patients seems much less than that previously described (Khan et al., 1979 and Wright et al., 1965).

Khan (1979), like Poole (1975), considers that the normal development and maintenance of joint structures is dependent upon joint movement such that patients with high spinal lesions and less limb mobility develop more frequent and more severe sacro-iliac change. (Wright (1965) hypothesised that these sacro-iliac changes are due to damage caused by severe mechanical stresses applied to the unsupported pelvis in paraplegia).

\section{Shoulders}

Wing (1983) described a group of 10 patients who, despite the strenuous activity of a 'swing through' gait on crutches, did not develop degenerative changes in the shoulders after a period of 7-8 years. Twelve of the 38 patients we studied developed degenerative changes. These changes were more common in the inactive rather than the active patient. These findings would seem to support the previously described finding that moderate joint activity protects the joint 
from degenerative changes rather than compromises the joint. Two patients who developed severe shoulder joint changes had underlying predisposing abnormalities: 1 patient had sustained bilateral fracture dislocation of the shoulder joint at the time of spinal injury, and the second patient had developed post-traumatic syringomyelia with a neuropathic shoulder.

\section{Conclusion}

In our retrospective study of 51 paraplegic patients who had been injured for 20 or more years, we found that the patients most prone to develop hip and sacroiliac joint degenerative changes are patients who have a high spinal cord injury and thus have the least effective muscle action to maintain synovial fluid circulation and production necessary for the normal sustenance of articular cartilage.

We have shown that a greater level of patient activity protects the hip and the shoulder joints.

In our group of patients we have found no correlation between the joint abnormalities and the incidence of urinary tract infection.

\section{Acknowledgements}

Our special thanks are due to Miss Jane McGibbon and Sister Norma Beer (from the Physiotherapy Department at Royal Perth (Rehabilitation) Hospital) for information supplied on level of activity undertaken by the patients reported in this study.

We thank Sir George Bedbrook for his encouragement and supervision of the work presented in this paper.

Our thanks also to Mrs J. Nixon, Mrs S. Mathie and Mrs M. Jackson for their help in preparing the manuscript.

\section{References}

ANDERSON JEM, BREIDAHL, PD 1981 Cartilage Atrophy Following Spinal Cord Damage.

Australasian Radiology XXV 1:98-103.

FredensBorg N, Nilsson BO E 1978 The Joint Space in Normal Hip Radiographs. Radiology 126:325-326.

KhAN MA, Kushner A, Freehafer AA 1979 Sacroiliac joint abnormalities in paraplegics. Annals of the Rheumatic Diseases 38:317-319.

Pool WH 1974 Cartilage Atrophy. Radiology 112:47-50.

Wing PC, Tredwell SJ 1983 The Weightbearing Shoulder. Paraplegia 21:107-113.

Wright V, Catterall RD, Cook JB 1965 Bone and Joint Changes in Paraplegic Men. Annals of the Rheumatic Diseases 24:419-431. 